\title{
Biotherapeutics and its applications in microbiology
}

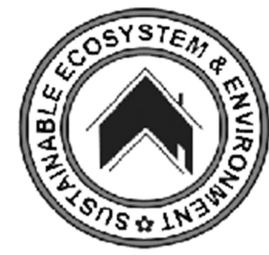

\author{
Kaur, N.肴, Chaudhary, V.
}

Received: 15.11 .2020

Revised: 06.01.2021

Accepted: 07.02.2021

\begin{abstract}
Biotherapeutics is a rapidly growing segment of the entire pharmaceutical industry that constitutes approximately onequarter of ongoing new drug endorsements. Monoclonal antibodies are a major part of these endorsements every year (mAbs). MAbs' non-clinical pharmacology and toxicology research compare with substance components during progression, since these biotherapeutics are extracted from an organic source, and to inspire a pharmacological reaction, the creature models must also have similar epitopes (focuses) as individuals. Biotherapeutic items (BTPs) are the quickest developing drugs in the pharmaceutical market. Despite their clinical achievement, the immunogenicity of BTPs keeps on being a significant concern. The subcutaneous (SC) course is to cultivate a passion for the organisation of biotherapeutics. Both monoclonal antibodies and various biotherapeutics are discussed. Medicine has been disrupted by biotherapeutic drugs (BPs), altering the way we treat a few processes. Comparative BPs (SBPs) are discussed here, also called biosimilars, including the assembly process and administrative viewpoints used. Monoclonal antibodies can apply synergistic antitumour impacts in blend with other immunomodulatory approaches, for example, chemotherapy, radiotherapy, directed treatment specialists, immunisations, or different immunomodulators. Probiotics have gotten profoundly perceived as enhancements for people and specifically for creatures given their gainful result on wellbeing improvement and prosperity support.
\end{abstract}

Key Words: Biotherapeutics, pharmaceutical, immunogenicity, probiotics, microbiology

\section{Introduction}

Biotherapeutics or Biologicals are drug therapy products that derive or manufacture the active agent from a biological source. These include recombinant proteins and hormones, monoclonal antibodies (mAbs), cytokines, growth factors, products for gene therapy, vaccinations, products for cell therapy, gene silencing/editing products, products for tissue engineering, and stem cell therapy (Dale et al., 2018). Biotherapeutics are the biological medicines that are produced from biological means, including recombinant DNA technology (Bonin-Debs et al., 2004). The important biologicals include the blood components, antibodies, microbiota, and human reproductive cells. These are therapeutic agents that, through recombinant DNA technology, biotechnological processing, and chemical synthesis using nucleotides or amino acids, are derived from living organisms or their products and involve genetically modified microbes (GMOs)

\section{Author's Address}

Department of Microbiology, College of Basic Sciences and Humanities, Punjab Agricultural University (PAU), Ludhiana, Punjab - 141004, India

E-mail.: navjotkaur778@gmail.com
(Mandell et al., 2015). Pharmaceuticals are, in addition to biotherapeutics, small molecule medicines made up of organic or metallic compounds that bind to proteins in the body, modifying their function and role in diseases. Via chemical synthesis, pharmaceuticals are developed and can act intracellularly and usually orally via aspirin, antibiotics, etc. The manufacturing cost is low and easy to replicate after patent expiration. Biotherapeutics are medications whose dynamic fixings are or are gotten from proteins, (for example, development hormone, insulin, antibodies) and different substances created by living life forms, (for example, cells, infections, and microorganisms) (Excipients, 2012). In the modern biopharmaceutical pipelines, natural therapy has reformed existing medication, adding new particles, such as monoclonal antibodies (mAbs), the largest biotherapeutic item (BP) (WHO 2009; Kay 2011; Roger 2010)- that have changed how we can treat some incessant conditions, including immune system sicknesses (ADs), etc. From a more extensive perspective, BPs have been labelled by the WHO as "drugs acquired from microorganisms, blood or other living tissues whose assembling 


\section{Navjot and Chaudhary}

strategies may incorporate one of the accompanying components: development of microorganisms, strains in various sorts of a substrate, utilisation of eukaryotic cells, organic substances extricated from tissues, including human, creature and plant tissues, and items acquired by recombinant DNA or hybridoma innovation, and the proliferation of microorganisms in incipient organisms or creatures, among others"(Pombo et al., 2009). That involves immunisations, allergens, antigens, hormones, cytokines, catalysts, human blood, and plasma subordinates, immunological serums, monoclonal immunoglobulins, antibodies, ageing agents, and in vitro determination reagents. Insulin, human development hormone, erythropoietin, granulocyte province animating part, IFN-an, and mAbs, among others, are economically available natural medications (Dranitsaris et al., 2011). A biotherapeutic product that is not identical to the original/reference biotherapeutic product as referred to in the WHO Guidelines should not be referred to as "like" or referred to as SBP.

An SBP has been characterised by WHO as "a BP which is comparable as far as quality, wellbeing, and adequacy to a previously authorised reference BP (RBP)"."In 2014, the World Health Assembly gave the mandate to the WHO to "update the 2009 Guidelines, taking into account developments in technology for the characterisation of biotherapeutic products. An assortment of terms has been utilised to reference these items, including the term 'biosimilars'. Second-age biopharmaceuticals are particles with a synthetic structure not quite the same as RBPs, adjusted to improve useful traits while safeguarding the instrument of activity (Kay 2011). Bio-better is recombinant protein tranquilise which, however, are strengthened over the first in a class comparable to a current biopharmaceutical. Bio-better focuses on a similarly permitted epitope as the promoted counteracting agent on account of antibodies but has been developed to have enhanced properties, such as advanced glycosylation profiles to boost effector capacities or a built Fc space to extend serum half-life (Beck 2011). Such biopharmaceuticals are not typically regarded as SBPs. Significant improvements in the production process (MP) of RBPs raise a degree of risk, such as that posed by SBP. For example, biotherapeutic products derived from recombinant
DNA invention have taken a serious step forward in the wellbeing and viability of useful peptides and proteins, interferons, interleukins, and growth factors, and have a successful record of treating various dangerous and incessant diseases. The United States Food and Drug Administration (FDA or USFDA) is a government agency of the Department of Health and Human Services. The FDA is responsible for the preservation and promotion of public goods by controlling and tracking food safety, tobacco products, dietary supplements, prescription and over-the-counter pharmaceuticals (medicines), vaccines, biopharmaceuticals, blood transfusions, medical supplies, electromagnetic radiation emitting devices (ERED), cosmetics, animal food \& feed and veterinary products (FDA 2015). The United States Food and Drug Administration (USFDA) has licensed 46 medicines, and out of these, 22 are approved as biotherapeutics. The approved biotherapeutics involves Monoclonal antibodies and these antibodies are typically designed with immunomodulatory function for cancer therapy. With the help of biotherapeutics, many patients are leading healthier lives as a result of biotherapeutic medicines, e.g. Insulin extracted from animals was used to treat diabetes. Biotherapeutics medicines play a role in the discovery and development of biomarkers which further helps in predicting the risk of cancer, diagnosing it, and indicating a potential effective course of treatment. The untreated autoimmune diseases such as Chron's disease and rheumatoid arthritis, which lead to early mortality have been diagnosed by the use of biotherapeutic medicines.

\section{Biotherapeutics}

The principal substance endorsed for remedial utilise was biosynthetic "human" insulin made using recombinant DNA technology (Clement et al., 2002). In certain cases referred to as rHI, Genentech was developed under the exchange name Humulin, but approved by Eli Lilly and Company, who manufactured and promoted it starting in 1982. Interferon alfa was initially endorsed in 1986 for the treatment of bushy cell leukaemia, preceded by other oncology and antiviral signs. Likewise in 1986, muromonabCD3, the main showcased monoclonal counter acting agent, was endorsed for the treatment of allograft dismissal. This murine monoclonal 
counter acting agent turned around intense renal dismissal in more prominent than $90 \%$ of cases, and it was the first promoted item produced using a deified mammalian cell combination (Becker and Reichert, 2007). In 1987, alteplase was affirmed for the treatment of intense myocardial areas of localised necrosis; other helpful catalysts with cardiovascular signs followed. Alteplase creation utilised deified Chinese hamster ovary cells $(\mathrm{CHO})$, further propelling the job of mammalian cell lines in the assembling of biotechnology items (Hristea and Khalili, 2000). From 1989 to 1991, the hematopoietic development factors erythropoietin, granulocyte settlement invigorating element (G$\mathrm{CSF}$ ), and granulocyte-macrophage province animating component (GM-CSF, delivered by recombinant yeast) were endorsed for advertising in the United States (Gouilleuxet al., 1995). These items treat frailty by decreasing transfusions or treat neutropenia by diminishing contaminations. The word propelled care restorative products (ATMPs) is used by the European Medicines Agency for medicines used by humans 'depending on the qualities, cells, or tissue engineering,' including quality treatment medicines, significant cell treatment prescriptions, and mixtures of tissuedesigned medicines. For example, gene-based and cell biologics are regularly at the cutting edge of biomedical research and may be used to treat a range of ailments for which there are no distinct drugs available. Biologics are handled in a few locations by separate pathways from other small particle drugs and clinical instruments. In 2003, the European Medicines Agency presented an adjusted pathway for biosimilars, named comparative organic therapeutic items. This pathway depends on an exhaustive exhibition of "equivalence" of the "comparable" item to a current affirmed product (EMA, 2008). The Patient Safety and Affordable Care Act of 2010 in the United States made a restricted endorsement route for natural products that are biosimilar to or compatible with an FDAauthorized reference organic product (Nick 2012; USFDA 2010). In 2009, the World Health Organization promoted the instructions that refer to the blueprint for advancing and assessing similar biotherapeutic products. IovanceBiotherapeutics is a biopharmaceutical creation situated in San Carlos, California. The organisation attempts to create tumour-infiltrating lymphocyte (TIL) treatments facing tumours (Garber, 2019; Adelman, 2019).

\section{Biotherapeutics proffering}

As seen, the word "biologics" can be used to refer to a wide variety of natural medicinal products. Nonetheless, as a rule, the expression "biologics" is utilised all the more prohibitively for a class of therapeutics (either affirmed or being developed) that are delivered by methods for natural procedures including recombinant DNA innovation (Von Schwerin et al., 2015). These drugs are normally one of three kinds, such as (1) Substances that are (about) indistinguishable from the body's key flagging proteins, (2) Monoclonal antibodies. Models are the erythropoietin protein animating blood-creation, or the hormone invigorating growth called (just) "development hormone" or human biosynthetic insulin and its analogs. They are like the antibodies used by the healthy human system to resist microscopic organisms and pathogens, but they are "specially crafted" and can then be rendered specifically to complement or square any given material in the body or to concentrate on a particular type of cell; the table below provides instances of such monoclonal antibodies for use in various ailments. (3) Receptor builds (combination proteins), typically dependent on a normally happening receptor connected to the immunoglobulin outline (Apgar et al., 2016). For this situation, the receptor furnishes the develop with point by point explicitness, while the immunoglobulin-structure bestows steadiness and other helpful highlights as far as pharmacology. A few models are recorded in the table beneath.

Biologics have profoundly affected numerous clinical fields as a class of medicines in this smaller sense, primarily rheumatology and oncology, but also cardiology, dermatology, gastroenterology, nervous system science, and others (Khalil and Huang, 2020). Biologics have included significant remedial options for the treatment of numerous diseases in the vast majority of these orders, including some for which no viable treatment has been available and others for which previously existing treatment has been lacking. In any event, the advent of biological therapeutics has also posed complex administrative problems (see below) and important pharmaco-economic issues, provided that the cost of biological therapies has been considerably higher than that of ordinary 


\section{Navjot and Chaudhary}

(pharmacological) medicines. This aspect was particularly important since various natural medicinal products are used for the treatment of incessant diseases, such as rheumatoid joint pain or provoking intestinal disease, or for the treatment of untreatable diseases during the rest of life, in any case. For the majority of patients each year, the cost of care with a run of the mill monoclonal immune response treatment for moderately simple signs is between EUR 7,000-14,000 for each patient. More established patients who get biological treatment for illnesses, for example, rheumatoid joint pain, psoriatic joint inflammation, or ankylosing spondylitis are at expanded hazard forever undermining disease, antagonistic cardiovascular occasions, and malignancy (Kerr, 2010).

Microbial cells (e.g. recombinant E. coli or yeast societies), mammalian cell lines (see cell culture), and plant cell societies (see plant tissue culture) and greenery plants can be supplied with biopharmaceuticals in bioreactors of various sizes, including photographic bioreactors (Decker et al., 2008). Cost of production (low-volume, highimmaculateness goods are attractive) and microbial sullying are key issues of concern (by microorganisms, infections, mycoplasma). The elective foundation of creation is being tried to incorporate entire plants (plant-made pharmaceuticals). Transgenic species, especially plants and creatures that have been hereditarily altered to produce drugs, are a conceivably disputable technique for generating biopharmaceuticals. This creation is a huge hazard for the financial specialist, because of creation disappointment or investigation from administrative bodies dependent on saw dangers and moral issues. Biopharmaceutical crops additionally speak to a danger of cross-pollution with non-built yields or harvests designed for the non-clinical purpose. The creation of a transgenic warm-blooded organism that can deliver the biopharmaceutical in its milk, blood, or pee is one possible way to deal with this breakthrough. When a creature is delivered, ordinarily utilising the pronuclear microinjection technique, it gets adequate to utilise cloning innovation to make extra posterity that conveys the greatly adjusted genome (Dove, 2000). ATryn was the main drug derived from the milk of a hereditary goat; however, the European Medicines Agency in February 2006 hindered the display of authorisation
(Phillip, 2006; Bhutiani and Ahamad, 2019). In June 2006, this option was modified, and approval was granted in August 2006.

\section{Mechanism of action of Monoclonal antibodies}

Monoclonal antibodies can be utilised as the bearer or homing gadget just as the effector warhead. They can be connected to drugs, poisons, radionucleotides, cytokines, or compounds for analytic or remedial purposes. They give prompt momentary insusceptibility against infections and different life forms that can be utilised as organic weapons. Many monoclonal antibodies are in clinical or preclinical turn of events, and blends or "mixed drinks" with various specificities are being examined. The immunological properties of the counteracting agent should be identified in detail for monoclonal antibodies, including its antigenic specificity, official supplementation, and any inadvertent reactivity or potentially cytotoxicity to human tissues that is unmistakable from the proposed goal. For monoclonal antibodies and other related immune response items coordinated at remote targets (for example bacterial, viral targets, and so on), a present moment (for example fourteen days term) wellbeing concentrate in one animal groups (with the selection of species legitimised by the support) can be thought of, no extra harmfulness considers, including regenerative poisonousness contemplates, are required.

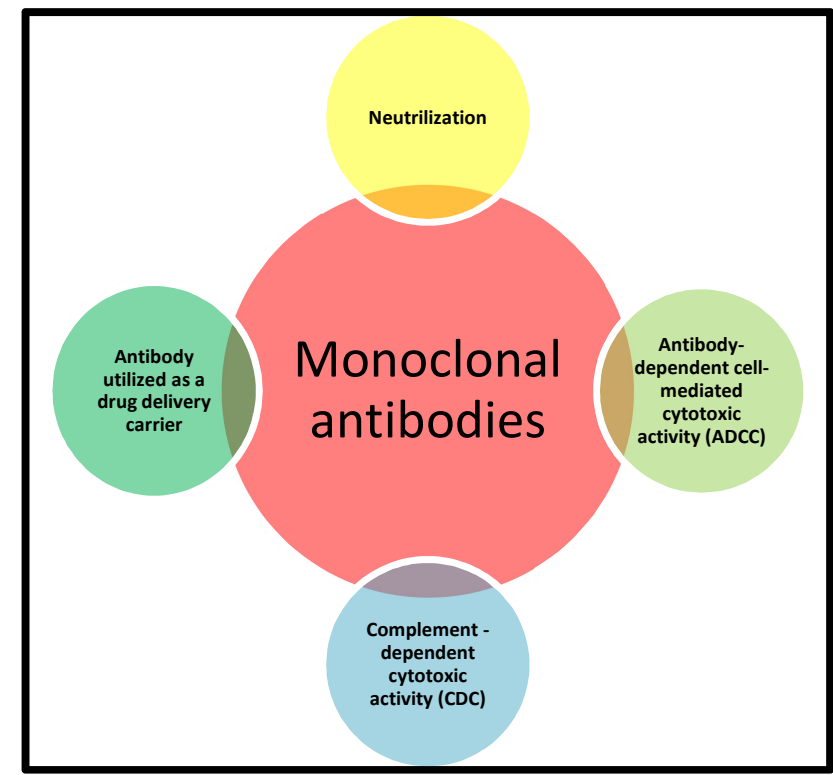

Figure 1. Therapeutic monoclonal antibodies act on target defected cells in four different ways. 
At the point when creature models of illness are utilised to get confirmation of guideline, a security evaluation can be incorporated to give data on potential objective related wellbeing viewpoints. Where this isn't achievable, suitable hazard relief methodologies ought to be embraced for clinical preliminaries. The capacity of certain antibodies to disturb flagging pathways associated with the upkeep of the dangerous phenotype has gotten boundless consideration. Be that as it may, the capacity of antibodies to start tumour-explicit resistant reactions has been less very much perceived. Here, we depict these components and talk about the potential for utilising antibodies to control the invulnerable host reaction to tumours. We centre around three instruments: ADCC, CDC, and the acceptance of versatile insusceptible reactions (Golay and Introna, 2012).

Neutralisation involves the therapeutic antibodies which help in blocking the pathophysiological function of their target molecules. These antibodies then bind to the ligand or receptor that has been shown on the surface of the cell and prevent the signalling pathway of the target. When signalling via these ligands or receptors in the tumour is blocked, cellular activity loss and proliferation inhibition occur after blocking the signalling pathway. (Shanahan et al., 2009).

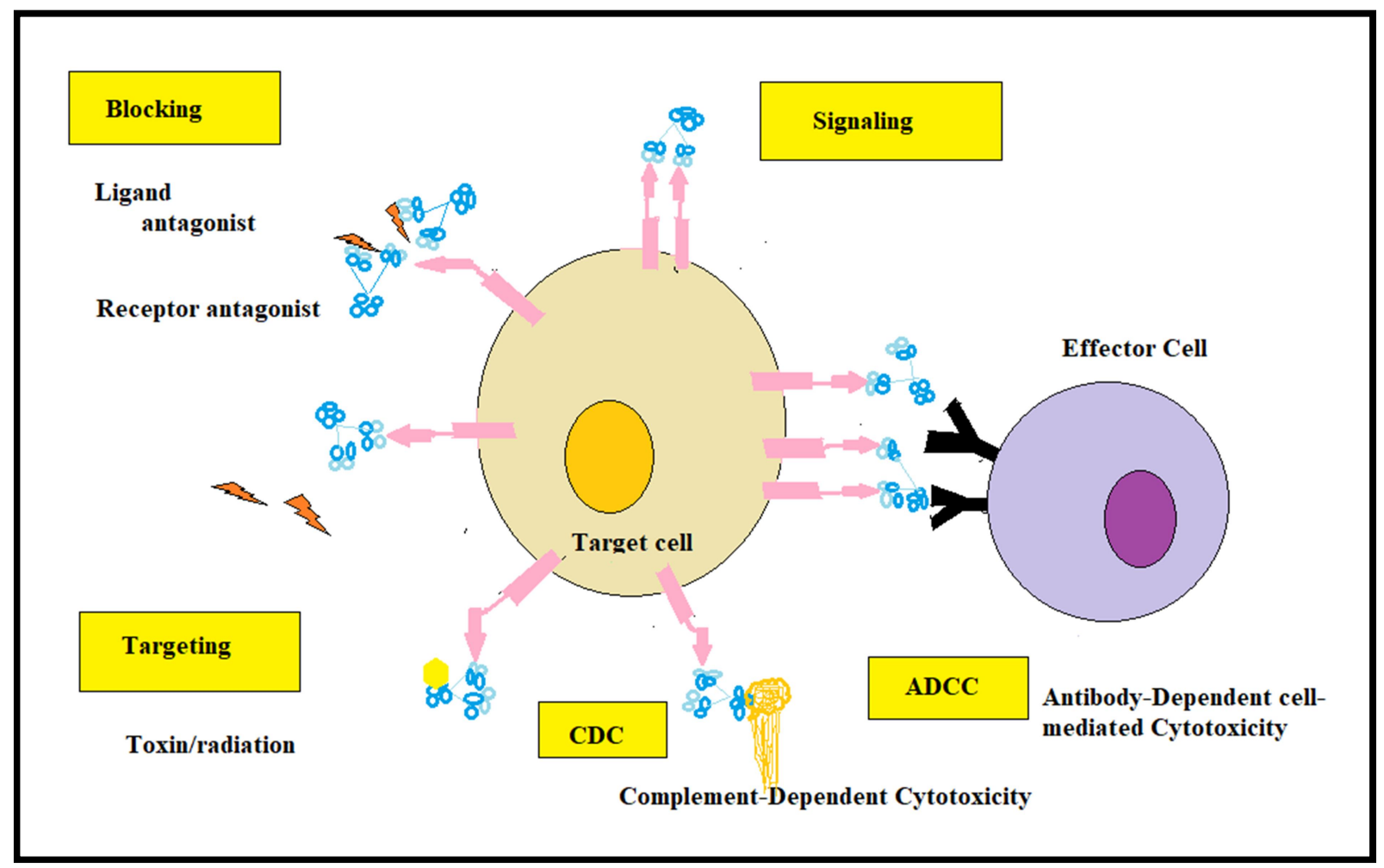

Figure 2. Mechanism of action of Monoclonal Antibodies

Antibody-dependent cell-mediated cytotoxic activity (ADCC)

In ADCC, an antibody's Fv binding domain binds to a particular antigen that is expressed on the surface of a target cell. The antibody can then convert immune effector cells (macrophages and NK cells) that represent multiple Fc-attached receptors and activate the immune effector cells to kill the target cell. In murine models and clinical preliminaries, a few research studies have identified the significance of Fc-Fc $\gamma \mathrm{R}$ connections for the in vivo antitumour effects of certain monoclonal antibodies. A simple paper suggested that trastuzumab and rituximab (Rituxan/Mabthera; Genentech/Roche/Biogen Idec) antitumour exercises were lower in Fc $\gamma \mathrm{R}$-insufficient mice than in wild-type mice (Clynes et al., 2000). Furthermore, the work of $\mathrm{Fc} \gamma \mathrm{R}$ in the antitumour reaction was supported by the finding that polymorphisms in the Fc $\gamma$ RIII consistency 
encoding, leading to higher Fc $\gamma$ RIII counteracting agent authority, are correlated with high rates of rituximab reaction in patients with follicular nonHodgkin lymphoma (Cartron, 2002). A separate study comparing clinical reactions to rituximab in patients with follicular lymphoma recommended that both FcyRIII and FcyRIIB had a role in the rituximab reaction (Weng and Levy, 2003). Later discoveries show that Fc $\gamma R$-encoding polymorphisms are associated with clinical reactions to various antibodies, including trastuzumab (Musolino, 2008) and cetuximab (Bibeau, 2009). It was found that patients with bosom malignancy who responded to trastuzumab with complete or fractional reduction had a higher capacity to interfere with trastuzumab in vitro ADCC than patients whose tumours had failed to respond to therapy. ADCC enhancement has been shown to guarantee the advancement of cuttingedge antibodies by $\mathrm{Fc}$ region modification. For instance, a CD19-explicit immune response with expanded Fc $\gamma$ RIIIA restricting partiality intervened essentially expanded ADCC contrasted with its parental counteracting agent and rituximab (Horton, 2008). The in vivo implantation of this high fondness counteracting agent proficiently cleared threatening B cells in cynomolgus macaques (Macacafascicularis) (Zalevsky, 2009).

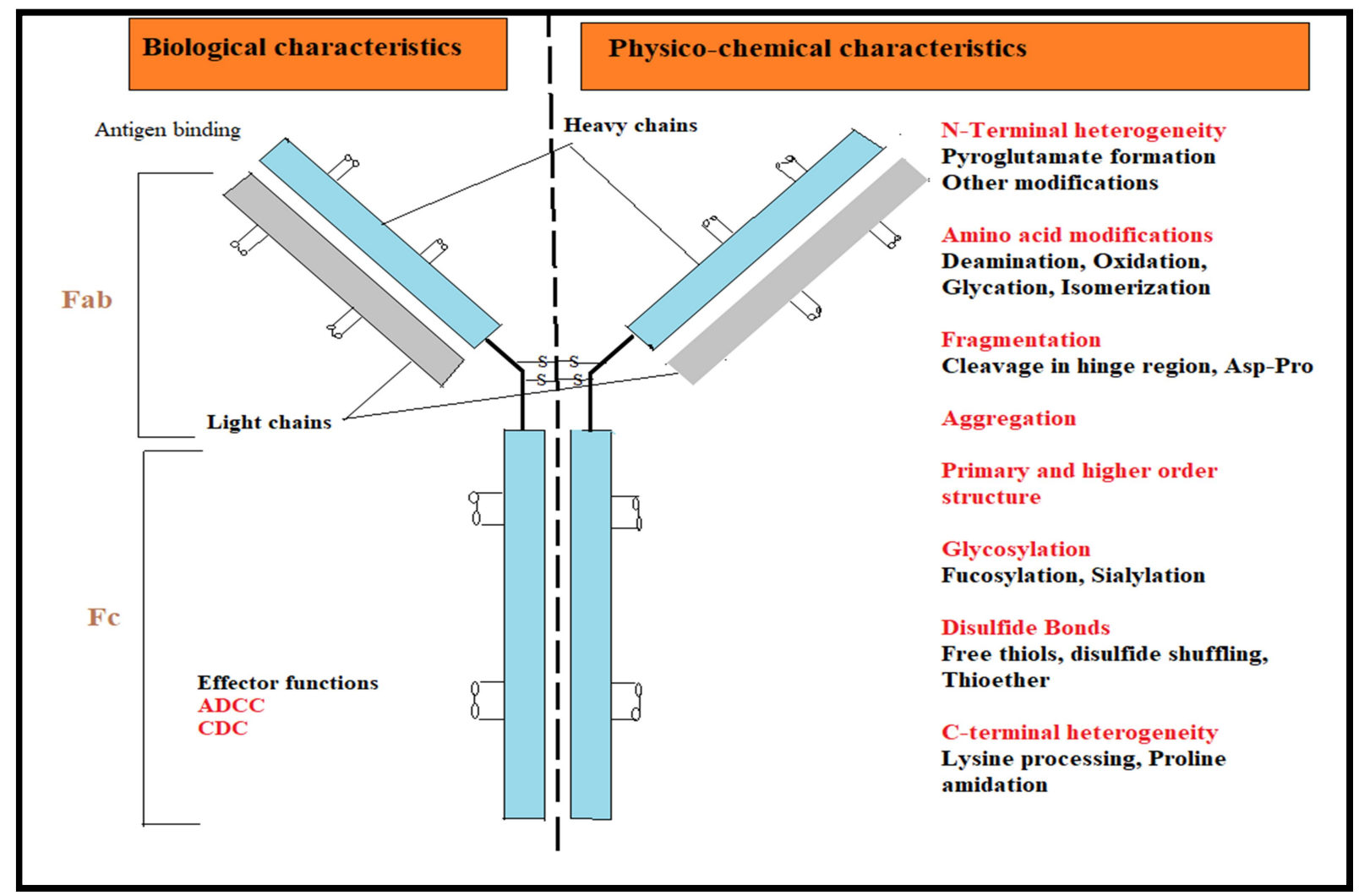

Figure 3. Structure and function of a generalised monoclonal antibody

Complement-dependent cytotoxic activity (CDC) host immune system and among the cancerIt is provoked when the $\mathrm{Cl}$ complex attaches to the developing therapeutic antibodies. Also, most antibody-antigen complex than the binding of the scientifically endorsed monoclonal antibodies that $\mathrm{C} 1$ complex with this complex triggers a cascade of interfere with ADCC initiate the supplement complement proteins. The activation of structure (Villamore et al., 2003). In any event, complement proteins, these activated types of inpatients with ceaseless lymphocytic leukaemia, protein attack on the target cell membrane resulting alemtuzumab that perceives CD52 communicated in target cell lysis, and the interactions between on developing $\mathrm{B}$ and $\mathrm{T}$ cells enacts human both ADCC and CDC include components of the supplement and has antitumour action, but it does 
not intercede with ADCC (Lundin, 2002). Also, the link between supplement enactment and remedial movement is suggested from concentrates with a few monoclonal antibodies clinically approved. For its in vivo adequacy, CD20-explicit monoclonal immune response rituximab was seen as needy to some degree by CDC. Rituximab antitumour insurance was nullified in C1q-lacking mice in a preclinical treatment model (Di Gaetano, 2003). In the xenograft model of human B cell lymphoma, supplementation was also shown to decrease the remedial function of rituximab (Cragg et al., 2004). The importance of rituximab-mediated $\mathrm{CDC}$ is supported by the evidence that genetic C1QA quality polymorphisms are correlated with clinical reactions to treatment with rituximab in patients with follicular lymphoma (Racila, 2008). Antitumour movement can be improved by streamlining immune response-based supplement exercises. For example, for the treatment of patients with constant lymphocytic leukaemia (CLL), the explicit CD20 counteracting agent of atumumab (Arzerra; Genmab/Glaxo SmithKline), which intervenes in enhanced CDC, was confirmed in 2009. Compared to rituximab, this entirely human counteracting agent connects an unexpected epitope with improved limiting energy and induces intense tumour cell lysis by improved initiation of the oldstyle supplement pathway (Coiffier 2008). The underlying analysis in patients with stubborn CLL revealed that of atumumab had a half-reaction rate, recommending greater adequacy than rituximab in patients with CLL, even though this higher reaction rate might not be the sole explanation for upgraded CDCs. A few tests show that both CDC and ADCC can add to the lysis of monoclonal neutraliserinitiated tumour cells. The relative clinical importance of each device, however, and whether these components are synergistic, substance-added, or aggressive, remains unclear. In a lymphoma mouse model, for example, supplement fatigue enhances NK cell actuation and ADCC along these lines, increasing the adequacy of the antibody (Wang, 2009).

Antibody utilised as a drug delivery carrier This is also known as missile therapy. Tumour targeting treatment that is "missile treatment", utilising a complex made out of a tumoursuppressive medication and an entire neutraliser against tumour cells is relied upon to turn into an appealing chemotherapy system."Missile treatment against tumour veins" by utilising explicit monoclonal antibodies against tumour endothelial cells as opposed to real tumour cells. The complex between antibodies to tumour vascular endothelial cells and against tumour medications can unreservedly get to the objective cells without worry for their vascular penetrability. These arrangements have displayed phenomenal enemies of tumour impacts for strong tumours. The antibodies are used as drug delivery carriers which are associated with radioisotopes, toxins, drugs, or cytokines. Then these conjugates play a role over traditional drugs in that cytotoxic agents can be administered to tumour tissues reliably and at higher local concentrations beyond causing any damage to normal cells (Bae and Park, 2011).

\section{Microbes used as biotherapeutic agents}

McFarlandand Elmer (1995) described

biotherapeutic specialists as living smaller scale life forms used to prevent or treat human infection by associating them with the host's normal microbial biology. Microscopic species or 'probiotics' have usually pointed to the treatment of various forms of gastrointestinal problems, in either case, more recently, hardly any studies have extended their usage towards vaginal contamination (Elmer et al., 1996; Sarkisov et al., 2000; Lenoir-Wijnkoop et al., 2007). Utilitarian nourishments containing biotherapeutic specialists have long been used for self-care and reciprocal medication from a general perspective (Sarkar, 2007). Microorganisms are used as a biotherapeutic agent due to their multipronged mechanism, the evolution of microbes against resistance, rate of replication, immune stimulation, genetic engineering.

\section{Multi-pronged mechanism}

Microorganisms rely upon a multi-pronged system for the endurance inside the various condition; for example, bacterial pathogens can beat have obstructions to contamination, including by infiltrating skin, withstanding stomach causticity, and conquer have protection component. Because of this, microorganisms can be designed to use against a specific pathogen rather than drugs (Bloch et al., 2013).

\section{Evolution of microbes against resistance}

Advancement can be utilised to broaden the adequacy of the microorganisms being created as therapeutics. Adjustment of microorganisms can 


\section{Navjot and Chaudhary}

likewise be utilised to react against the specific pathogen (Medzhitov 2007).

\section{Rate of replication}

Remedial organisms imitate just inside the host at the site of contamination. Along these lines, they enhance the portion of this inside condition where it is required while maintaining a strategic distance from reactions from this treatment somewhere else in the body. The pace of replication can be related to the pathogen; for example, change in portion mirrors the measure of a tumour inside the tissue (Harper et al., 2014).

\section{Immune stimulation and Genetic Engineering}

Organisms can animate the safe host framework to assault pathogens. They can invigorate both inborn and versatile invulnerable reactions in facilitated design for progressively compelling treatment (Chaplin 2010) and can change hereditarily to improve their wellbeing and adequacy in the treatment of contaminations.
As a biotherapeutic agents, specific carbohydrates provides a therapeutic properties to control the vaginal infections that are caused by Candida albicans.
As antibacterial agents, biotherapeutics are used to treat severe Clostridium difficle associated diseases (CDAD).

\section{Applications of Biotherapeutics}

Lactobacillus species can be used as a

biotherapeutic agent for the activation of host

immune system to treat the cancer.
The 4 species of probiotic bacteria

(L.acidophilus, L. reuteri, L. casei,

Bifidobacterium animalis)are ued to treat

mucosal and systemic candidiasis.

Figure 4. Applications of Biotherapeutics in microbiology

\section{Vaginal infections}

As for the basic management of vaginal diseases, biotherapeutic drugs may have a positive effect (Elmer et al., 1996; Sarkisov et al., 2000) explicitly by prebiotic starches (Sutherland et al., 2008) and probiotic microorganisms (Maggi et al., 2000; Reid et al., 2003). For this reason, both polysaccharides and oligosaccharide starches can be used (Rousseau et al., 2005; Bou-Antoun 2008; Coste et al., 2012; Linhares et al., 2013; Hou et al., 2014). Enthusiasm for probiotics as practical nutrition segments offers the ability to control the gut microbiota (Shanahan et al., 2009). Current studies have, however, advanced prebiotic applications in conditions other than the gut (Gibson and Roberfroid 1995; Roberfroid 2007) and include oral cleanliness (Tester and Al- Ghazzewi 2011; Maitra et al., 2013), safe skin (Al-Ghazzewi and Tester 2010), and vaginal wellbeing (Sutherland et al.,
2008; Tester et al., 2012). The lactic corrosive microscopic organisms use vaginal glycogen under the hormonal influence to manufacture lactic corrosives (Nasioudis et al., 2015), which are responsible for controlling the acidic (hostile to pathogenic) vaginal state (Turovskiy et al., 2011). Even though the vaginal environment is all things considered acidic occasions, the movement of amylase at low $\mathrm{pH}$ is reduced, yet to distinguishable levels, which can contribute to maintaining the production of Lactobacillus at a constrained but supported pace (Spear et al., 2015). Relevant nonendogenous starches offer an elective restorative way to deal with microbial infection regulation by repressing the vaginal epithelial cell bond of pathogens (Al-Ghazzewi and Tester 2014), and this complements any 'prebiotic' function. In this regard, mannose-rich starches are efficient (Tester and Al- 
Ghazzewi, 2016). Oral and topical probiotic methods of coping with the vaginal infection have been studied by (Reid et al., 2001, 2003; Anukam et al., 2006; Petricevic and Witt 2008; Hemmerling et al., 2010). To treat (and probably forestall repeats of) molested vaginal yeast diseases, common solutions have long been used. These are remembered for particular yoghurts, where the rich items of the velvety acidic lactic corrosive microscopic organisms provide comfort (Neri et al., 1993; Hantoushzadeh et al., 2012). Lactobacillus delbrueckii subsp. is usually a probiotic microorganism in yoghurts. Streptococcus thermophilus and Streptococcus bulgaricus. Moreover, other lactobacilli and bifidobacteria are often found every so often within 'advanced' bioyoghurt during or in the wake of processed yoghurt, for example. The utilisation of yoghurts depends chiefly on the thought that the probiotics inside them can act as biotherapeutic factors and develop inside the vaginal depression.

\section{Cancer treatment}

Lactobacillus species can utilise as a biotherapeutic specialist for initiation of host invulnerable framework to treat the malignant growth. Because of the bounty and periodicity of the twodimensional crystalline S-layer, parts of the S-layer of Lactobacillus species can be adjusted (Sleytr et al., 2001). After the alteration, Lactobacillus microorganisms can prompt cytokines that pull in resistant cells, animate a safe reaction against the tumour. A large number of these antibodies are explicit for antigens communicated by the tumour itself. Antibodies associated with radioactive isotopes or chemotherapeutic drugs have demonstrated beneficial viability mainly in haematological malignancies, while unconjugated antibodies based on development factor receptors are routinely used for the treatment of nonleukemic malignancies, such as epidermal development factor receptor (EGFR, otherwise called ERBB1) and human epidermal development factor receptor 2 (HER2, otherwise called ERBB2 or NEU) (Henson et al., 2017). Notwithstanding antibodies targeting tumour antigens, tumour microenvironment-focused antibodies moderate tumour development by upgrading invulnerable host reactions to tumour-related antigens or by abridging the tumour-related factors generated in the tumour stroma.

\section{Clostridium difficile infections}

Clostridium difficile is a Gram-positive, sporeforming, and anaerobic gastrointestinal (GI) bacterium that can cause a variety of diseases such as anti-microbial bowel looseness (AAD), Pseudomembranous colitis, and poisonous megacolon (Bartlett 2006; Brazier 2008; Ozaki et al., 2004; Hurley et al., 2002). C. difficile infections (CDI) is the digestive organ's incendiary state represented by the looseness of the intestines and the presence of specific plaques and neutrophils throughout the intestinal lumen (Adams and Mercer 2007; Bartlett et al., 1978). Antibodies for CDI have been being developed for more than two decades. A C-inactivated formalin. When administered by nasal, peritoneal, and subcutaneous courses, difficult culture filtrate was found to guarantee hamsters (Torres et al., 1995). Giannasca et al. (1999) discovered C. inactivated. Hamsters were protected by difficult poison (toxoid) when given via the intramuscular and rectal courses. All the more as of late, intraperitoneal infusion of an adjuvanted toxoid B immunisation has been appeared to keep the illness from a poison B-positive, poison A-negative strain of C. difficile in hamsters however didn't forestall colonisation (Siddiqui et al., 2011).

$\mathrm{CDI}$ is a disease for which counteraction techniques ought to be exceptionally viable, given that both biotherapeutic and insusceptible methodologies are indicating a guarantee. Every one of these methodologies has points of interest and impediments regarding the rate of assurance, the term of security, and likely expense. Biotherapeutics and monoclonal or other immunologic methodologies are probably going to give just transient assurance, though immunisations need to give all the more long-haul security if they are to be successful. Almost certainly, an injectable antidote poison antibody will be the first to be affirmed for human use and should be promptly reachable given the effectively-recognised poison antigen targets and the related knowledge with profoundly fruitful toxoid immunisation for lockjaw. Given the mucosal idea of the C. difficile disease, my predisposition is toward the achievement of an inevitable mucosal immunisation that will probably be utilised in grown-ups, yet in kids too. The improvement of new biotherapeutics and mucosal immunisations is reliant on revealing 
new information on the connection between the $\mathrm{C}$. difficile creature, the two spores, and vegetative cells, and the mucosal interface in the host. Remembered for the last is a depiction of both inborn and versatile mucosal safe reactions. The standpoint for the avoidance of CDI is certain. The open door managed by new revelations at the hostpathogen interface will lead, more likely than not, to better biotherapeutic and immunisation/immunologic systems for the anticipation of CDI (Gerding, 2012).

\section{Infectious diseases}

In almost all persons, Candida occupies the gastrointestinal (GI) tract, and a substantial portion of the diseases, including Candida, are endogenously procured from the GI tract. Through the unblemished gastrointestinal mucosa, Candida may pass into the circulation system and spread to instinctive organs, triggering fundamental candidiasis, especially in essentially sick patients (Demirel et al., 2013). Candida excess is encouraged by the disruption of ordinary physiological obstructions, such as gastric sharpness and annoyances of the indigenous colon microflora. The most commonly known site of contamination within the GI tract is the throat. As a shrewd pathogen that postpones ulcer recovery and irritates the condition, Candida may be linked to gastric ulcers (Brzozowski et al., 2005). For certain decades, foundational antifungal specialists have been effectively used to forestall mucosal just as obtrusive parasitic diseases. In any case, because of the medication symptoms (sickness, regurgitating, and loose bowels), and the potential rise of safe strains, antifungal prophylaxis has not been effective. Polyenes (nystatin and amphotericin B) and azoles (fluconazole, itraconazole, voriconazole) are commonly used antifungals. Strangely, Candida's biofilm age is considerably more impervious to both of these opposing antifungals and their planktonic allies (Sardi et al., 2013). The utilisation of probiotic microorganisms against microbial diseases has risen as an elective restorative procedure for Candida contaminations taking into account the impediments of the as of now accessible anti-microbials. Probiotics are described as live microorganisms that offer wellbeing benefits to the host when managed or expended in satisfactory quantities. Microscopic species that have a position in the genera
Lactobacillus and Bifidobacterium and, to a lesser extent, Enterococcus, Streptococcus, and Saccharomyces have been commonly used as probiotics for an extensive timeframe in food supplements (Saarela et al., 2000). A sheltered probiotic should be of the human root, without natural and transmissible anti-microbial obstruction qualities. The useful necessities of a probiotic incorporate corrosive and bile resistances, satisfactory adherence and colonisation on epithelial surfaces, invulnerable incitement, and adversarial movement against pathogens (Saarela et al., 2000).

In remedial terms, in different organ frameworks of the human body, probiotics are considered to reduce Candida diseases and are generally seen as beneficial for general wellbeing. Probiotics, for example, can combat bowel looseness (primarily in young people) and decrease lactose bias and side effects of inflammatory gut infections (Saarela et al., 2000). Also, probiotic microscopic organisms have been explored for their potential for forestalling diseases, for example, colorectal malignancy (Shida et al., 2013), controlling pulse (Khalesi et al., 2014), and stifling cholesterol levels (Jones et al., 2013). The combination of probiotics with traditional treatment alternatives is assumed to yield improved outcomes and disease resolution in different areas, with only a small increase in the cost of treatment (Saarela et al., 2000; Li et al., 2014; Martinez et al., 2009). Also, there are records of possible antiviral effects of probiotics, primarily against respiratory viral pathogens, in people of any age (Lehtoranta et al., 2014). The antifungal effect of polymicrobial mixes of probiotics against human Candida albicans isolated from oral depression, GI tract, and the genitourinary tract has been shown in several in vitro studies (Comman et al., 2014; Hasslof et al., 2010; Vilela et al., 2015). A significant marvel seen in some of these studies was the formation of hydrogen peroxide by probiotics that threaten candidate development (Strus et al., 2005; Verdenelli et al., 2014), immunocompromised children, especially preterm neonates with low birth weight, were the objective population of various tests evaluating the effectiveness of probiotics against candidate colonisation of the GI tract (Demirel et al., 2013; Manzoni et al., 2006; Romeo et al., 2011; Roy et al., 2014). The choice of a readily available 72 
probiotic-bound drink containing Lactobacillus casei and Bifidobacterium breve was to minimise the predominance of oral Candida in solid individuals (Mendonca et al., 2012).

\section{Future perspectives}

Conveyance choices of Biotherapeutics are at present constrained (primary courses are intravenous and subcutaneous) and the objective Mediated Disposition may prompt Nonlinearity. The assembling is fundamentally progressively unpredictable, and a basic factor in wellbeing and adequacy subsequently fabricating changes must be deliberately surveyed (Comparability). Items can prompt immunogenicity where the body mounts an insusceptible reaction to the item. This is particularly valid for items that contain different species segments (for example, giving human protein to creature's protein to creatures for wellbeing considers). The species particularity may restrain standard preclinical models for wellbeing testing. The monoclonal counteracting agent is presently the best quality level biopharmaceutical, however different configurations, some of which despite everything depend on the monoclonal immune response as the premise, are being developed or have just been popularised. Regardless of whether they will dislodge the monoclonal immune response, later on, we will need to sit back and watch, yet the signs are that the monoclonal neutraliser still has a solid future. What ties the present and future biopharmaceuticals together are their intricacy and requirement for intensive characterisation to guarantee wellbeing and adequacy. Also, SBPs improve improved access to BPs, which benefits impacted individuals and advances competition within the biotechnology showcase. In any case, the present guidelines on SBPs must support the section of those which guarantee the quality, security, and adequacy, as

\section{References}

Adams, S. D. and Mercer, D. W. 2007. Fulminant Clostridium difficile colitis. Current Opinion in Critical Care, 13: 45055.

Adelman, J. 2019. New Navy Yard lab and office complex planned for Calif. cell-therapy research firm Iovance. The Philadelphia Inquirer. was expressed by WHO in their SBPs rule: "The elaboration of the information prerequisites and contemplations for the permitting of BPs will encourage the advancement of and overall access to biotherapeutics of guaranteed quality, wellbeing and viability at increasingly reasonable costs". Similar to any definitions of live organisms, the capacity for the determination of healthy strains, alterability, and passability on delayed use, as well as pathogenic potential in immuno-traded patients, are integrated into various issues that require further review.

\section{Conclusion}

Biologics give safe arrangements in numerous territories of neglected clinical need, and their development is relied upon to proceed, particularly in the regenerative treatment and customised medication. In any case, their significant expenses, a stricter administrative condition, and the rise of biosimilars present difficulties to this advancement. Biotherapeutics are an essential and significant piece of current medication for the treatment and avoidance of genuine ailments and illnesses. The utilisation of biotherapeutics brings about the finding of diseases brought about by multi-sedate safe microscopic organisms, treatment of malignant growth, vaginal contaminations. Various sorts of bearers are being used for the vehicle of biotherapeutics at the site of the disease rapidly for focusing on the pathogen including Monoclonal antibodies treatment, bacteriophage treatment and utilisation of parts of living microorganisms in late medications. For new techniques to include an increasingly prescient software to assess and screen possible unfriendly drug responses of mAbs for explicit patients before and during clinical preliminaries and reseller exchange endorsement, there are difficulties and potentially open doors.

Al-Ghazzewi, F. H. and Tester, R. 2014. Inhibition of the adhesion of Escherichia coli to human epithelial cells by carbohydrates. Bioactive Carbohydrates and Dietary Fibre, 4 :1-5.

Al-Ghazzewi, F. H. and Tester, R. F. 2010. Effect of konjac glucomannan hydrolysates and probiotics on the growth of the skin bacterium Propionibacterium acnes in vitro. International Journal of Cosmetic Science, 32: 139-142. 


\section{Navjot and Chaudhary}

Anukam, K. C., Osazuwa, E., Osemene, G. I., Ehigiagbe, F., Bruce, A. W. and Reid, G. 2006. Clinical study comparing probiotic Lactobacillus GR-1 and RC-14 with metronidazole vaginal gel to treat symptomatic bacterial vaginosis. Microbes Infection, 8: 2772-2776.

Anukam, K., Osazuwa, E., Ahonkhai, I., Ngwu, M., Osemene, G., Bruce, A. W. and Reid, G. 2006. Augmentation of antimicrobial metronidazole therapy of bacterial vaginosis with oral probiotic Lactobacillus rhamnosus GR-1 and Lactobacillus reuteri RC-14: randomized, double-blind, placebo controlled trial. Microbes Infection, 8: 14501454.

Apgar, J. F., Wong, J., Phennicie, R., Briskin, M. and Burke, J. M. 2016. Abstract B34: Quantitative systems pharmacology and immunotherapy: accelerating lead generation and optimization of a PD-1 $x$ TIM-3 biotherapeutic in immuno-oncology.

Bae, Y. H. and Park, K. 2011. Targeted drug delivery to tumors: myths, reality and possibility. Journal of controlled release, 153(3): 198.

Bartlett, J. G. 2006. Narrative review: the new epidemic of Clostridium difficile-associated enteric disease. Annals of Internal Medicine, 145: 758-764.

Bartlett, J. G., Chang, T. W., Gurwith, M., Gorbach, S. L. and Onderdonk, A. B. 1978. Antibiotic-associated pseudomembranous colitis due to toxin-producing clostridia. The New England Journal of Medicine, 298: 531-534.

Beck, A. 2011. Biosimilar, biobetter and next generation therapeutic antibodies. Monoclonal Antibodies, 3(2): 107110

Becker, H. and Reichert, J. M. 2007. Muromonab-CD3 (Orthoclone OKT3). Handbook of therapeutic antibodies, 905-940.

Bhutiani, R. and Ahamad, F. 2019. A case study on changing pattern of agriculture and related factors at Najibabad region of Bijnor, India. In: Contaminants in Agriculture and Environment: Health Risks and Remediation. Edited by Vinod Kumar, Rohitashw Kumar, Jogendra Singh and Pankaj Kumar (ISBN: 978-81-942017-0-0). DOI: 10.26832/AESA-2019-CAE-0158-018, pp 237-247.

Bibeau, F. 2009. Impact of Fc $\gamma$ RIIa-Fc $\gamma$ RIIIa polymorphisms and KRaS mutations on the clinical outcome of patients with metastatic colorectal cancer treated with cetuximab plus irinotecan. International Journal of Clinical Oncology, 27: 1122-1129.

Bloch, E. F., Schultz, R. D. and Turner, W. 2013. Mini-review: Probiotics and disease prevention in different host systems. Microbiology Research Journal International, 42-57.
Bonin-Debs, A. L., Boche, I., Gille, H. and Brinkmann, U. 2004. Development of secreted proteins as biotherapeutic agents. Expert opinion on biological therapy, 4(4): 551558.

Bou-Antoun, S. 2008. Compositions that aim to promote the development and growth of a beneficial vaginal microflora. European Patent EP, 2303300.

Brazier, J. S. 2008. Clostridium difficile: from obscurity to superbug. British Journal of Biomedical Science, 65: 3944.

Brzozowski, T., Zwolinska-Wcislo, M.and Konturek, P. C. 2005. Influence of gastric colonization with Candida albicans on ulcer healing in rats: effect of ranitidine, aspirin and probiotic therapy. Scandinavian Journal of Gastroenterol, 40: 286-296.

Cartron, G. 2002. Therapeutic activity of humanized anti-CD20 monoclonal antibody and polymorphism in $\mathrm{IgG} \mathrm{Fc} \mathrm{receptor}$ Fc $\gamma$ RIIIa gene. Blood, 99: 754-758.

Chaplin, D. D. 2010. Overview of the immune response. Journal of Allergy and Clinical Immunology, 125(2): 323.

Clement, S., Still, J. G., Kosutic, G. and McAllister, R. G 2002. Oral insulin product hexyl-insulin monoconjugate 2 (HIM2) in type 1 diabetes mellitus: the glucose stabilization effects of HIM2. Diabetes technology \& therapeutics, 4(4): 459-466.

Clynes, R. A., Towers, T. L., Presta, L.G. and Ravetch, J. V. 2000. Inhibitory Fc receptors modulate in vivo cytoxicity against tumor targets. Nature Medicine, 6: 443-446.

Coiffier, B. 2008. Safety and efficacy of ofatumumab, a fully human monoclonal anti-CD20 antibody, in patients with relapsed or refractory b-cell chronic lymphocytic leukemia: a phase 1-2 study. Blood, 111: 1094-100.

Coman, M. M., Verdenelli, M. C. and Cecchini, C. 2014. In vitro evaluation of antimicrobial activity of Lactobacillus rhamnosus IMC 501®, Lactobacillus paracasei IMC 502® and SYNBIO ${ }^{\circledR}$ against pathogens. Journal of Applied Microbiol, 117: 518-527.

Coste, I., Judlin, P., Lepargneur, J. P. and Bou-Antoun, S. 2012. Safety and efficacy of an intravaginal prebiotic gel in the prevention of recurrent bacterial vaginosis: a randomised double-blind study. International Journal of Obstetrics and Gynecology, 147867.

Cragg, M.S.and G.lennie, M. J. 2004. Antibody specificity controls in vivo effector mechanisms of anti-CD20 reagents. Blood, 103: 2738-2743.

Dale, E. and Johnson. 2018. Biotherapeutics: Challenges and opportunities for predictive toxicology of monoclonal 
antibodies.International Journal of Molecular Sciences, doi:10.3390/ijms19113685.

Decker, E. L. and Reski, R. 2008. Current achievements in the production of complex biopharmaceuticals with moss bioreactors. Bioprocess and Biosystems Engineering, 31(1): 3-9.

Demirel, G., Celik, I. H., Erdeve, O., Saygan, S., Dilmen, U. and Canpolat, F. E. 2013. Prophylactic Saccharomyces boulardii versus nystatin for the prevention of fungal colonization and invasive fungal infection in premature infants. European Journal of Pediatrics, 172: 1321-1326.

Di Gaetano, N. 2003. Complement activation determines the therapeutic activity of rituximab in vivo. Journal of Immunology, 171: 1581-1587.

Dove, A. 2000. Milking the genome for profit. Nature Biotechnology, 18(10): 1045-1048.

Dranitsaris, G., Amir, E. and Dorward, K. 2011. Biosimilars of biological drug therapies. Drugs, 71(12): 1527-1536.

Elmer, G. W., Surawicz, C. M. and McFarland, L. V. 1996. Biotherapeutic agents: a Neglected modality for the treatment and prevention of selected intestinal and vaginal infections. Journal of the American Medical Association, 275: 870-876.

EMA 2008. Questions and answers on biosimilar medicines (similar biological medicinal products). Dc. Ref. EMEA/74562/2006 Rev. 1

Garber Ken 2019. "Pursuit of tumor-infiltrating lymphocyte immunotherapy speeds up". Nature Bio, 37:969-977

Gerding, D. N. 2012. Clostridium difficile infection prevention: biotherapeutics, immunologics, and vaccines. Discovery medicine, 13(68): 75-83.

Giannasca, P. J., Zhang, Z. X., Lei, W. D., Boden, J. A., Giel, M. A., Monath, T. P. and Thomas, W. D. 1999. Serum antitoxin antibodies mediate systemic and mucosal protection from Clostridium difficile disease in hamsters. Infection and Immunity, 67(2): 527-538.

Golay, J. and Introna, M. 2012. Mechanism of action of therapeutic monoclonal antibodies: promises and pitfalls of in vitro and in vivo assays. Archives of biochemistry and biophysics, 526(2):146-153.

Gouilleux, F., Pallard, C., DusanterFourt, I., Wakao, H., Haldosen, L. A., Norstedt, G. and Groner, B. 1995. Prolactin, growth hormone, erythropoietin and granulocyte macrophage colony stimulating factor induce MGF Stat5 DNA binding activity. The EMBO journal, 14(9): 20052013.
2012. Comparative efficacy of probiotic yoghurt and clindamycin in treatment of bacterial vaginosis in pregnant women: a randomised clinical trial. Journal of MatenalFetal Neonatal Medicine, 25: 1021-1024.

Harper, D. R., Burrowes, B. H. and Kutter, E. M. 2014. Bacteriophage: therapeutic uses. $\boldsymbol{e L S}$.

Hasslöf, P., Hedberg, M., Twetman, S. and Stecksen-Blicks, C. 2010. Growth inhibition of oral mutans streptococci and candida by commercial probiotic lactobacilli-an in vitro study. BMC Oral Health, 10-18.

Hemmerling, A., Harrison, W., Schroeder, A., Park, J., Korn, A., Shiboski, S., Foster-Rosales, A. and Cohen, C. R. 2010. Phase 2a study assessing colonization efficiency, safety, and acceptability of Lactobacillus crispatus CTV-05 in women with bacterial vaginosis. Sexually Transmitted Diseases, 37: 745-750.

Henson, E., Chen, Y. and Gibson, S. 2017. EGFR family members' regulation of autophagy is at a crossroads of cell survival and death in cancer. Cancers, 9(4): 27.

Hou, W., Han, L., Li, M., Chen, J. and Chen, Y. 2014. Effectiveness evaluation of alginate oligosaccharides antibacterial gel for bacterial vaginosis. Journal of Life Sciences, 11: 528-531.

Hristea, I. and Khalili, V. 2000. Alteplase (TPA) for clotted dialysis catheters. 668-669.

Hurley, B. W. and Nguyen, C. C. 2002. The spectrum of pseudomembranous enterocolitis and antibiotic-associated diarrhea. Archievesof Internal Medicine, 162: 2177-2184.

Jones, M. L., Tomaro-Duchesneau, C., Martoni, C. J. and Prakash, S. 2013. Cholesterol lowering with bile salt hydrolase-active probiotic bacteria, mechanism of action, clinical evidence, and future direction for heart health applications. Expert OpinonBiological Therapy, 13: 631642.

Kay, J. 2011. Biosimilars: a regulatory perspective from America. Arthritis Research \& Therapy, 13(3): 112.

Kerr, L. D. 2010. The use of biologic agents in the geriatric population. Journal of Musculoskeletal Medicine, 27: 175-180.

Khalesi, S., Sun, J., Buys, N. and Jayasinghe, R. 2014. Effect of probiotics on blood pressure: a systematic review and meta-analysis of randomized, controlled trials. Hypertension, 64: 897-903.

Khalil, H. and Huang, C. 2020. Adverse drug reactions in primary care: a scoping review. BMC Health Services Research, 20(1): 5.

Hantoushzadeh, S., Golshahi, F., Javadian, P., Khazardoost, S., Lehtoranta, L., Pitkaranta, A. and Korpela, R. 2014. Probiotics Aram, S., Hashemi, S., Mirarmandehi, B. and Borna, S. in respiratory virus infections. European Journal of 
Clinical Microbiology \& Infectious Diseases, 33: 1289 1302.

Lenoir-Wijnkoop, I., Sanders, M. E., Cabana, M. D., Caglar, E., Corthier, G., Rayes, N., Sherman, P. M. and Timmerman, H. M. 2007. Probiotic and prebiotic influence beyond the intestinal tract. Nutrition Reviews, 65: 469-489.

Li, D., Li, Q. and Liu, C. 2014. Efficacy and safety of probiotics in the treatment of Candida-associated stomatitis. Mycoses, 57: 141-146.

Linhares, L. M., Kanninen, T., Orfanelli, T., Jayaram, A., Doulaveris, G. and Witkin, S. S. 2013. The vaginal microbiome: new findings bring new opportunities. Drug Development Research, 74: 360-364.

Lundin, J. 2002. Phase II trial of subcutaneous antiCD52 monoclonal antibody alemtuzumab $($ Campath $1 \mathrm{H})$ as firstline treatment for patients with b-cell chronic lymphocytic leukemia (b-CLL). Blood, 100: 768-773.

McFarland, L. V. and Elmer, G. W. 1995. Biotherapeutic agents: past, present and future. MicroecolTher. 23: 46 73.

Maggi, L., Mastromarino, P., Macchia, S., Brigidi, P., Pirovano, F., Matteuzzi, D. and Conte, U. 2000. Technological and biological evaluation of tablets containing different strains of lactobacilli for vaginal administration. European Journal of Pharmaceuticals and Biopharmaceuticals, 50: 389-395.

Maitra, A., Rollins, M., Tran, L., Al-Ghazzewi, F. and Tester, R. 2013. Prebiotic konjac glucomannan hydrolysate reduces Streptococcus mutans in oral biofilms. International Association for Dental Research (IADR) Abstracts. March 20-23, Seattle, Washington, USA.

Mandell, D. J., Lajoie, M. J., Mee, M. T., Takeuchi, R., Kuznetsov, G., Norville, J. E. and Church, G. M. 2015. Biocontainment of genetically modified organisms by synthetic protein design. Nature, 518 (7537): 55-60.

Manzoni, P., Mostert, M. and Leonessa, M. L. 2006. Oral supplementation with Lactobacillus casei sub species rhamnosus prevents enteric colonization by Candida species in preterm neonates: a randomized study. Clinical Infectious Disease, 42: 1735-1742.

Martinez, R. C., Franceschini, S. A. and Patta, M. C. 2009. Improved treatment of vulvovaginal candidiasis with fluconazole plus probiotic Lactobacillus rhamnosus GR-1 and Lactobacillus reuteri RC-14. Letters in Applied Microbiology, 48: 269-274.

Medzhitov, R. 2007. Recognition of microorganisms and activation of the immune response. Nature, 449 (7164): 819-826.

Musolino, A. 2008. Immunoglobulin G. fragment C receptor polymorphisms and clinical efficacy of trastuzumab-based

\section{Chaudhary}

therapy in patients with HER-2/ neu-positive metastatic breast cancer. Journal of Clinical Oncology, 26: 17891796.

Nasioudis, D., Beghini, J., Bongiovanni, A. M., Giraldo, P. C., Linhares, I. M. and Witkin, S. S. 2015. a-Amylase in vaqginal fluid: association with conditions favourable to dominance of Lactobacillus. Reproductive Sciences, 22: 1393-1398.

Neri, A., Sabah, G. and Samra, Z. 1993. Bacterial vaginosis in pregnancy treated with yoghurt. Acta Obstetricia Gynecologica Scandinavica, 72: 17-19.

Nick, C. 2012. The US Biosimilars Act: Challenges Facing Regulatory Approval. Pharmacy Medical, 26(3): 145-152.

Ozaki, E., Kato, H. and Kita, H. 2004. Clostridium difficile colonization in healthy adults: transient colonization and correlation with enterococcal colonization. Journal of Medical Microbioloh, 53: 167-172.

Petricevic, L. and Witt, A. 2008 . The role of Lactobacillus caseirhamnosusLcr 35 in restoring the normal vaginal flora after antibiotic treatment of bacterial vaginosis. British Journal of Obstetrics Gynaecology, 115: 1369-1374.

Phillip, B. C. Jones. 2006. European Regulators Curdle Plans for Goat Milk Human Antithrombin.

Pombo, M. L., Di Fabio, J. L. and Cortes Mde, L. 2009. Review of regulation of biological and biotechnological products in Latin American and Caribbean countries. Biologicals, 37(5): 271-276.

Racila, E. 2008. A polymorphism in the complement component $\mathrm{C} 1 \mathrm{qa}$ correlates with prolonged response following rituximab therapy of follicular lymphoma. Clinical Cancer Research, 14: 6697-6703.

Reid, G., Beuerman, D., Heinemann, C. and Bruce, A. W. 2001. Probiotic Lactobacillus dose required to restore and maintain a normal vaginal flora. Federation of European Microbiological Societies Immunology \& Medical Microbiology, 32: 37-41.

Reid, G., Charbonneau, D., Erb, J., Kochanowski, B., Beuerman, D., Poehner, R., Poehner, R. and Bruce, A. W. 2003. Oral use of Lactobacillus rhamnosus GR-1 and L. fermentum RC-14 significantly alters vaginal flora: randomised, placebo-controled trial in 64 healthy women. Federation of European Microbiological Societies Immunology \& Medical Microbiology, 35:131-134.

Roberfroid, M. B. 2007. Prebiotics: the concept revisited. Journal of Nutrition, 137: 830-837.

Roger, S. D. 2010. Biosimilars: current status and future directions. Expert Opinion on Biological Therapy, 10(7): 1011-1018. 
Romeo, M. G., Romeo, D. M. and Trovato, L. 2011. Role of probiotics in the prevention of the enteric colonization by Candida in preterm newborns: incidence of late-onset sepsis and neurological outcome. Journal of Perinatology, 31: 63-69.

Rousseau, V., Lepargneur, J. P., Roques, C., Remaud-Simeon, M. and Paul, F. 2005. Prebiotic effects of oligosaccharides on selected vaginal lactobacilli and pathogenic microorganisms. Anaerobe, 11: 145-153.

Roy, A., Chaudhuri, J., Sarkar, D., Ghosh, P. and Chakraborty, S. 2014. Role of enteric supplementation of probiotics on late-onset sepsis by Candida species in preterm low birth weight neonates: a randomized, double blind, placebocontrolled trial. North American Journal of Medical Sciences, 6: 50-57.

Saarela, M., Mogensen, G., Fonden, R., Matto, J. and MattilaSandholm, T. 2000. Probiotic bacteria: safety, functional and technological properties. Journal of Biotechnology, 84: 197-215.

Sardi, J. C., Scorzoni, L., Bernardi, T., Fusco-Almeida, A. M. and Mendes Giannini, M. J. 2013. Candida species: current epidemiology, pathogenicity, biofilm formation, natural antifungal products and new therapeutic options. Journal of Medical Microbiology, 62(1): 10-24.

Sarkar, S. 2007. Functional foods as self-care and complementary medicine. Nutration Food Science, 37: $160-167$.

Sarkisov, S. E., Krymshokalova, Z. S., Kafarskaia, L. I. and Korshunov, V. M. 2000. The use of biotheraputic agent Zhlemilk for correcting the microflora in bacterial vaginosis. Journal of Microbiology, Epidemiology and Immunobiology, 1: 88 -90.

Sarkisov, S.E., Krymshokalova, Z.S., Kafarskaia, L.I. and

Shanahan, F., Stanton, C., Ross, P. and Hill, C. 2009. Pharmabiotics: bioactive from mining host-microbedietary interactions. Functional Food Reviews, 1: 20-25.

Shida, K. and Nomoto, K. 2013. Probiotics as efficient immunopotentiators: translational role in cancer prevention. Indian Journal of Medical Research, 138: 808-814.

Siddiqui F, O'Connor, J. R., Nagaro, K., Adam,Cheknis., Sambol, S. P., Vedantam, G., Gerding, D. N.and Johnson, S. 2011. Vaccination with parenteral toxoid B protects hamsters against lethal challenge with toxin A-negative, toxin B-positive Clostridium difficile but does not prevent colonization. Journal of Infectious Diseases, epub ahead of print.

Sleytr, U. B., Sára, M., Pum, D. and Schuster, B. 2001. Characterization and use of crystalline bacterial cell surface layers. Progress in Surface Science, 68(7-8): 231-278.
Spear, G. T., McKenna, M., Landay, A. L., Makinde, H., Hamaker, B., French, A. L. and Lee, B. H. 2015. Effect of $\mathrm{pH}$ on cleavage of glycogen by vaginal enzymes. PLoS One, 10, e 0132646.

Strus, M., Kucharska, A., Kukla, G., Brzychczy-Wloch, M., Maresz, K. and Heczko, P. B. 2005. The in vitro activity of vaginal Lactobacillus with probiotic properties against Candida. Infectious Diseases in Obstetrics and Gynecology, 13: 69-75.

Sutherland, A., Tester, R., Al-Ghazzewi, F., McCulloch, E. and Connolly, M. 2008. Glucomannan hydrolysate (GMH) inhibition of Candida albicans growth in the presence of Lactobacillus and Lactococcus species. Microbial Ecology in Healthand Disease, 20: 127-134.

Tester, R. F. and Al-Ghazzewi, F. H. 2011. A preliminary study of the synbiotic effects of konjac glucomannan hydrolysates (GMH) and lactobacilli on the growth of the oral bacterium Streptococcus mutans. Nutrition and Food Science, 41: 234-237.

Tester, R. F. and Al-Ghazzewi, F. H. 2016. Beneficial health characteristics of native and hydrolysed konjac (Amorphophalus konjac) glucomannan. Journal of the Science of Food and Agriculture, doi: 10.1002/jsfa.7571.

Tester, R., Al-Ghazzewi, F., Shen, N., Chen, Z., Chen, F., Yang, J., Zhang, D. and Tang, M. 2012. The use of konjac glucomannan hydrolysates to recover healthy microbiota in infected vaginas treated with an antifungal agent. Beneficial Microbes, 3: 61-66.

Torres, J. F., Lyerly, D. M., Hill, J. E. and Monath, T. P. 1995. Evaluation of formalin-inactivated Clostridium difficile vaccines administered by parenteral and mucosal routes of immunization in hamsters. Infection and Immunity, 63 (12): 4619-4627.

Turovskiy, Y., SutyakNoll, K. and Chikindas, M. L. 2011 The aetiology of bacterial vaginosis. Journal of AppliedMicrobiology, 110: 1105-1128.

Verdenelli, M. C., Coman, M. M., Cecchini, C., Silvi, S., Orpianesi, C. and Cresci, A. 2014. Evaluation of anti pathogenic activity and adherence properties of human Lactobacillus strains for vaginal formulations. Journal of AppliedMicrobiology, 116: 1297-1307.

Vilela, S. F., Barbosa, J. O. and Rossoni, R. D. 2015. Lactobacillus acidophilus ATCC 4356 inhibitsbiofilmformationbyC.albicansandattenuatestheexper imentalcandidiasis in Galleria mellonella. Virulence, 6: 2939.

Villamor, N., Montserrat, E. and Colomer, D. 2003. Mechanism of action and resistance to monoclonal antibody therapy. In Seminars in oncology, 30 (4): 424433. 


\section{Navjot and Chaudhary}

Von Schwerin, A., Stoff, H. and Wahrig, B. 2015. Biologics, a history of agents made from living organisms in the twentieth century. Routledge.

Wang, S. Y. 2009. Depletion of the C3 component of complement enhances the ability of rituximabcoated target cells to activate human NK cells and improves the efficacy of monoclonal antibody therapy in an in vivo model. Blood, 4: 5322-5330.
Weng, W. K. and Levy, R. 2003. Two immunoglobulin G. fragment $\mathrm{C}$ receptor polymorphisms independently predict response to rituximab in patients with follicular lymphoma. Journal of Clinical Oncology, 21: 3940-3947.

Zalevsky, J. 2009. The impact of Fc engineering on an antiCD19 antibody: increased Fc $\gamma$ receptor affinity enhances bcell clearing in nonhuman primates. Blood, 11. 3735-3743. 